\title{
Fluid biomarkers in frontotemporal dementia: past, present and future
}

\author{
Imogen Joanna Swift (D) ,' Aitana Sogorb-Esteve, ${ }^{1,2}$ Carolin Heller (D) ,' \\ Matthis Synofzik, ${ }^{3,4}$ Markus Otto (1D) ,' Caroline Graff,, 6 , Daniela Galimberti (D) , 8,9 \\ Emily Todd, ${ }^{2}$ Amanda J Heslegrave, ${ }^{1}$ Emma Louise van der Ende (D) , ${ }^{10}$ \\ John Cornelis Van Swieten (D) , ${ }^{10}$ Henrik Zetterberg, ${ }^{1,11}$ Jonathan Daniel Rohrer (D) ${ }^{2}$
}

\begin{abstract}
- Additional material is published online only. To view, please visit the journal online (http://dx.doi.org/10.1136/ jnnp-2020-323520).

For numbered affiliations see end of article.
\end{abstract}

\section{Correspondence to} Dr Jonathan Daniel Rohrer, Dementia Research Centre, Department of Neurodegenerative Disease, UCL Queen Square Institute of Neurology, London WC1N 3BG, UK; j.rohrer@ucl.ac.uk

Received 5 August 2020 Revised 3 October 2020 Accepted 3 October 2020 Published Online First 13 November 2020

Check for updates

(c) Author(s) (or their employer(s)) 2021. No commercial re-use. See rights and permissions. Published by BMJ.

To cite: Swift IJ, Sogorb-

Esteve A, Heller C, et al. J

Neurol Neurosurg Psychiatry

2021:92:204-215.

\section{ABSTRACT}

The frontotemporal dementia (FTD) spectrum of neurodegenerative disorders includes a heterogeneous group of conditions. However, following on from a series of important molecular studies in the early 2000s, major advances have now been made in the understanding of the pathological and genetic underpinnings of the disease. In turn, alongside the development of novel methodologies for measuring proteins and other molecules in biological fluids, the last 10 years have seen a huge increase in biomarker studies within FTD. This recent past has focused on attempting to develop markers that will help differentiate FTD from other dementias (particularly Alzheimer's disease (AD)), as well as from non-neurodegenerative conditions such as primary psychiatric disorders. While cerebrospinal fluid, and more recently blood, markers of AD have been successfully developed, specific markers identifying primary tauopathies or TDP-43 proteinopathies are still lacking. More focus at the moment has been on non-specific markers of neurodegeneration, and in particular, multiple studies of neurofilament light chain have highlighted its importance as a diagnostic, prognostic and staging marker of FTD. As clinical trials get under way in specific genetic forms of FTD, measures of progranulin and dipeptide repeat proteins in biofluids have become important potential measures of therapeutic response. However, understanding of whether drugs restore cellular function will also be important, and studies of key pathophysiological processes, including neuroinflammation, lysosomal function and synaptic health, are also now becoming more common. There is much still to learn in the fluid biomarker field in FTD, but the creation of large multinational cohorts is facilitating better powered studies and will pave the way for larger omics studies, including proteomics, metabolomics and lipidomics, as well as investigations of multimodal biomarker combinations across fluids, brain imaging and other domains. Here we provide an overview of the past, present and future of fluid biomarkers within the FTD field.

\section{INTRODUCTION}

The frontotemporal dementia (FTD) spectrum encompasses a group of conditions that overlap in their clinical, neuroanatomical, genetic and pathological features. ${ }^{1}$ Clinically, FTD can be divided into a behavioural form (behavioural variant frontotemporal dementia (bvFTD)), a language variant (primary progressive aphasia (PPA)) and a motor presentation (either FTD with amyotrophic lateral sclerosis (FTD-ALS) or an atypical parkinsonian disorder). Neuroanatomically, the FTD spectrum is characteristically associated with dysfunction and neuronal loss in the frontal and temporal lobes, but more widespread cortical, subcortical, cerebellar and brainstem involvement is now recognised. Genetically, around a third of FTD is familial with autosomal dominant mutations in three genes accounting for most of the inheritance: progranulin $(G R N)$, chromosome 9 open reading frame 72 (C9orf72) and microtubule-associated protein tau $(M A P T) .^{2}$ Lastly, pathologically, cellular inclusions containing abnormal forms of tau, TDP-43 or FET proteins are found in the majority of people with an FTD syndrome. The interaction between clinical phenotype, neuroanatomy, genotype and pathology is complex (figure 1) and means that FTD can be hard to diagnose (particularly its specific pathological form during life) and difficult to track over time.

One way that researchers have aimed to solve some of these outstanding issues in the FTD field has been to develop fluid biomarkers, and there has been a growing literature in recent years investigating new cerebrospinal fluid (CSF) or blood measures in people with FTD. This review aimed to set out what has been done so far, where we are at present and what we still need to achieve in the future within the FTD fluid biomarker research world. This is particularly important in a time when potential therapies have now been developed and clinical trials have started.

\section{CLASSIFICATION AND USE OF BIOMARKERS IN FTD}

Fluid biomarkers, measured typically in CSF, serum or plasma (box 1) using a variety of different techniques (box 2), are objective indicators of normal or pathological biological processes or pharmacological responses to a therapeutic intervention. In FTD, biomarkers can be classified in a number of ways:

- Diagnostic, including distinguishing FTD versus non-neurodegenerative disorders and FTD versus Alzheimer's disease (AD) or other 


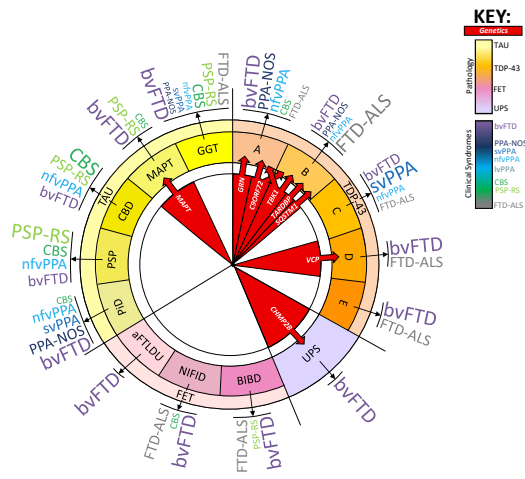

Figure 1 Clinical-pathological-genetic correlations in the FTD spectrum. The innermost part of the circle depicts the genetic causes of FTD. The middle part depicts the different pathological forms: PiD, PSP, CBD, MAPT, GGT, UPS, BIBD, NIFID and aFTLDU. The outermost part of the circle represents the clinical diagnoses associated with each pathology - the largest font being the most common syndrome, the medium font being syndromes less commonly seen and the smallest font being rare phenotypes. aFTLDU, atypical FTLD with ubiquitin inclusions; ALS, amyotrophic lateral sclerosis; BIBD, basophilic inclusion body disease; bvFTD, behavioural variant FTD; CBD, corticobasal degeneration; CBS, corticobasal syndrome; FTD, frontotemporal dementia; GGT, globular glial tauopathy; Iv, logopenic variant; MAPT, pathology associated with MAPT mutations; nfv, nonfluent variant; NIFID, neuronal intermediate filament inclusion disease; NOS, not otherwise specified; PiD, Pick's disease; PPA, primary progressive aphasia; PSP, progressive supranuclear palsy; RS, Richardson's syndrome; sv, semantic variant; UPS, ubiquitin-proteasome system.

dementias, as well as identifying the particular pathological form of FTD.

- Prognostic, allowing prediction of likely disease course and survival.

- Staging, including particularly for the genetic forms of FTD, whether someone is presymptomatic, nearing symptom onset (proximity markers) or phenoconverting.

- Therapeutic response, including showing target engagement and efficacy, as well as pharmacokinetic and pharmacodynamic responses.

Biomarkers can also provide an insight into the underlying pathophysiology of FTD and, in the context of clinical trials, can offer a direct experimental medicine approach to understanding molecular mechanisms through measurement of biofluids preand post-intervention. While some pathways are specific to one or other pathogenetic form of FTD, studies in recent years have particularly highlighted the importance of loss of synaptic integrity, lysosomal dysfunction and neuroinflammation as major underlying mechanisms across the FTD spectrum.

\section{DIFFERENTIATING FTD FROM OTHER DEMENTIAS AND FROM NON-DEGENERATIVE DISORDERS}

In the neurodegenerative biomarker field, much effort has been made in being able to differentiate AD from other dementias. Many of these studies have included an FTD cohort as a comparator group. Until recently, the focus has been on (usually combined) measures of tau (both 'total' and phosphorylated forms) and amyloid-beta (A $\beta$ ) in CSF. For example, a raised tau/A 342 ratio identifies those with likely pathological AD (eg, Paterson $e \mathrm{al}^{3}$ ) in comparison with those with likely FTD where the ratio is lower. Clinically, this is helpful in identifying atypical presentations of $\mathrm{AD}$, both in bvFTD (where such markers are an
Box 1 Biosamples

Cerebrospinal fluid (CSF) surrounds the brain and spinal cord, coming into direct contact with the extracellular space. It is therefore the most effective biological fluid to measure biochemical changes in brain tissue. However, in recent years, there has been an increasing focus on biomarkers in blood (plasma or serum), which forms a less invasive and more accessible alternative. Unfortunately, studies of blood-based biomarkers involve overcoming a number of challenges ${ }^{\mathrm{s1}}$ : first, the marker needs to be able to cross the blood-brain barrier (BBB), and if the marker is non-specific to the central nervous system, there is a risk of peripheral contamination; second, numerous other blood-based proteins and heterophilic antibodies, which are higher in blood than CSF, are likely to interfere with measurements; and finally, preanalytical factors, such as food intake or diurnal variation, need to be considered as these may be more relevant in blood. Fortunately, recent technological developments, such as Single molecule array (Simoa) technology (detailed further in box 2), allow for more sensitive assays, eliminating many of these challenges. One other potential way of improving peripheral identification of neuronally derived biomarkers is through the measurement of proteins within exosomes. ${ }^{\mathrm{s2}}$ These are small extracellular vesicles, released by cells, including within the brain, and able to cross the BBB, suggesting that measurement of their content in blood (as well as in CSF) may well represent central nervous system processes..$^{53}$ Technology is now available to extract neuronal exosomes within blood, ${ }_{1}^{\text {S4 }}$ making them an important prospect for future studies. Lastly, other body fluids poorly studied as potential sources of biomarkers in frontotemporal dementia are urine ${ }^{55}$ or saliva, ${ }^{\mathrm{S6}}$ and future studies would benefit from investigating these further.

exclusionary diagnostic criterion) and PPA, where the logopenic variant is usually an $\mathrm{AD}$ rather than FTD disorder pathologically. More recent studies have investigated blood-based markers of $\mathrm{AD}$, showing that plasma phosphorylated tau- 181 and tau- 217 are raised in AD but not FTD (apart from those with specific MAPT mutations that lead to accumulation of paired helical filament tau pathology similar to $\mathrm{AD}$, for example, $\mathrm{R} 406 \mathrm{~W}$ and $\mathrm{V} 337 \mathrm{M}){ }^{45}$

As an aside, one interesting point of note that has arisen from these studies has been the finding that $A \beta$ species, including $\mathrm{A} \beta 38, \mathrm{~A} \beta 40$ and sAPP $\beta$, are commonly lowered in FTD compared with controls, ${ }^{6}$ with the reason for this remaining unclear at present.

As well as difficulties in differentiating FTD from AD clinically on occasion, there can also be problems with differentiating FTD from non-neurodegenerative disorders at times, including those with primary psychiatric disorders. Recent studies have shown that changes in non-specific markers of degeneration (such as neurofilament light chain (NfL) protein (box 3)) may be helpful in this setting. ${ }^{7}$ However, more work needs to be done here as such markers are low in concentration when the speed of disease progression is also low, decreasing the ability to individually distinguish a non-degenerative neuropsychiatric disorder from a slowly progressive degenerative condition, a particularly important distinction in those that fit the phenotype of the so-called FTD phenocopy syndrome. 


\section{Box 2 Measurement techniques}

There are several platforms offering assays for biomarker measurement and discovery:

- ELISA is the classical technique to measure proteins in fluids. In the most common format, target-specific antibodies bind to the sample proteins, and a secondary antibody linked to an enzyme recognises the matched antibodies (a socalled sandwich ELISA). The conjugated enzyme can create a chromogenic or fluorescent reaction when exposed to a chemical substrate, with the amount of antigen present directly correlated to the intensity of the colour change. It is a quantitative technique that has been widely used in the biomarker field, although it usually only allows one analyte per assay and the detection range is inferior to other highsensitivity techniques (on the $\mathrm{pM}$ to $\mathrm{nM}$ range).

- Mesoscale Discovery (MSD) is a high-throughput platform to measure proteins, increasing the sensitivity of conventional ELISAs, measuring the levels of single or multiple targets within a single, small-volume sample. Unlike conventional ELISAs, detection antibodies can be directly conjugated to SULFO-TAG to generate electrochemiluminescent signals that have a voltage-dependent activation.

- Luminex is a high-throughput screening technology (on the picogram scale) that consists of a bead-based methodology in which each bead presents a different colour code and each one is conjugated with an antibody against a specific analyte. It can be very useful to develop and measure biomarker panels of up to 80 different analytes from a single microplate well, reducing the sample volume needed. With this platform, it is possible to measure not only protein but also RNA and DNA.

- Single molecule array or Simoa is an ultrasensitive immunoassay in two different versions. The conventional antibody-based ELISA in a planar array format and a bead-based platform in which each antibody-coated bead binds to a single molecule of analyte and then is analysed separately from the rest, offering a high sensitivity and wide detection range (on the $\mathrm{fM}$ to $\mathrm{pM}$ scale). The assay format allows multiplexing of up to 11 analytes and provides the possibility to develop home brew assays. A recently described upgrade of the technology might even allow for subattomolar quantification. $^{\mathrm{S7}}$

- The SomaScan platform is based on slow off-rate modified aptamers. Aptamers are short, single-stranded DNA or RNA molecules that can selectively bind to a specific target, including proteins, peptides, carbohydrates, small molecules, toxins and even live cells. In the SomaScan Platform, these aptamers bind to tertiary structures of the targets that are then quantified by standard DNA techniques such as microarrays or quantitative PCR ( $q P C R)$. The platform allows for the creation of a library of specific aptamers with high sensitivity for particular targets (from fM to pM concentrations).

- Proximity Extension Assay technology (Olink) consists of DNA oligonucleotide tags linked to a pair of matched antibodies that both bind to a target protein. When the matched antibodies come in close proximity on binding to the target protein, the DNA tags will hybridise; a DNA duplex will form; and then that sequence is amplified by qPCR. The platform provides a wide library of matched antibodies with high sensitivity and specificity for their targets.

Continued
Box 2 Continued

- In the Single Molecule Counting (SMC) platform, the antibody-antigen sandwich complexes, originating from either beads or plates, are broken up and only the fluorescently labelled detection antibody is counted one by one using a laser beam that excites the fluorophore. A digital event is counted if the fluorescence reaches above the threshold of the background. At higher concentrations, it is difficult to separate all events, and a switch can be made to use the total sum of all emitted photons as readout for the signal, allowing for a high dynamic range (on the $\mathrm{fM}$ to $\mathrm{pM}$ scale).

- Mass spectrometry is usually used for the discovery of new biomarkers, but targeted quantitative assays are also available, for example, through parallel reaction monitoring. It is the most specific technique but involves long development of each assay, and the instruments are expensive and require a high level of expertise. The technology is often used when establishing a reference method where analytical specificity is key, in biomarker discovery projects, or to measure small molecules, such as drugs, lipids and metabolites. Targeted protein biomarker panels are now getting increasingly common as well.

\section{DIAGNOSING PATHOGENETIC FORMS OF FTD DURING LIFE Genetic FTD}

FTD is genetic in around one third of people, with expansions in C9orf72 the most common cause and usually tested for through repeat-primed PCR and confirmed with Southern blotting. Mutations in the other common causes, GRN and MAPT, as well as the rarer genetic causes TBK1, TARDBP, VCP, FUS, CHMP2B, $S Q S T M 1$ and $U B Q L N 2$, are commonly tested for through either a targeted next-generation sequencing panel or increasingly commonly through exome or genome sequencing. However, for two of the major genetic causes of FTD (GRN mutations and C9orf72 expansions), there are specific biomarkers that can identify people who carry pathogenic mutations.

\section{Progranulin}

The majority of pathogenic variants in GRN are frameshift, nonsense or splice site mutations and cause haploinsufficiency, resulting in reduced levels of progranulin protein (figure $2 \mathrm{~A}$ ). This is measurable in both blood and CSF, although the majority of studies have been performed in blood. While initial studies showed a very high sensitivity and specificity (both $>95 \%$ ) with a cut-off of $61.5 \mathrm{ng} / \mathrm{mL}$ (measured in plasma by the Adipogen assay), ${ }^{8}$ a more recent study suggests a higher cut-off of $71.0 \mathrm{ng}$ $\mathrm{mL}^{9}$ (sensitivity $98.1 \%$, specificity $98.5 \%$ ). Levels seem to be low from the earliest they have been measured (late teens), with the assumption that they are decreased from birth, and with levels relatively constant over time (at least over 4 years in one study). ${ }^{9}$ In general, this makes measuring progranulin levels in blood a highly accurate (and less expensive) way of detecting a pathogenic mutation prior to (more expensive) genetic screening, as well as a way of confirming likely pathogenicity in splice site or missense variants (the latter more commonly being risk factors rather than directly pathogenic). Levels in controls are very variable (eg, from below the cut-off point to $>250 \mathrm{ng} / \mathrm{mL}$ in plasma) with the presence of a metabolic syndrome, autoimmune disease, obesity and cancer affecting levels as well as certain genetic factors (GRN rs5848, SORT1 rs646776 and possibly 
Box 3 Neurofilament light chain (NfL): diagnosis, prognosis and staging of frontotemporal dementia (FTD)

Neurofilaments are intracellular filaments found in the central and peripheral nervous systems. They can be found in different assemblies, including NfL, neurofilament medium chain (NfM) and neurofilament heavy chain (NfH). All of them function as elastic assemblies that help maintain cell shape ${ }^{58}$ and in neurons, this action controls axonal diameter, modulating the response to stimuli. When there is neuronal damage or axonal injury, they can leak and be found in the cerebrospinal fluid (CSF). Of all the neurofilaments, NfL has been the most studied. Initial studies in FTD within CSF showed that NfL was raised in many patients with different phenotypes. ${ }^{59}$ More recently, the use of an ultrasensitive assay on the Simoa platform has allowed accurate measurement of NfL within blood (with high correlation with CSF concentrations). Studies have shown that concentrations reflect how fast the disease is progressing (ie, disease intensity) ${ }^{510-512}$ and are a measure of survival in most FTD phenotypes ${ }^{513,514}$ but not all (eg, semantic variant PPA ${ }^{515,516}$ ). Diagnostically, while concentrations are variable and overlap with other neurodegenerative disorders, ${ }^{517} \mathrm{NfL}$ concentrations are nonetheless higher than in primary psychiatric disorder mimics of FTD. ${ }^{518}$ Furthermore, concentrations rise sharply just prior to symptom onset in genetic FTD as presymptomatic mutation carriers phenoconvert (figure 2C). ${ }^{513,519}$ In summary, the measurement of NfL offers a number of opportunities in the FTD biomarker field. First, as a diagnostic marker, it helps to differentiate FTD in the clinical setting from a nonneurodegenerative disorder. Second, as a marker of proximity to symptom onset, its baseline measurement is likely to predict progression to clinical symptoms in presymptomatic mutation carriers. Third, its measurement during the symptomatic period provides an indication of how fast the disease is progressing and therefore likely prognosis. Lastly, similar to other diseases, the ability to decrease NfL within the context of a disease-modifying trial is likely to be a positive indication of a decrease in the rate of neurodegeneration. ${ }^{520}$ Fewer studies have investigated NfM or $\mathrm{NfH}$ : levels of both are increased in $\mathrm{FTD}_{1}^{, 521,522}$ although their utility seems less than for NfL, except when there is associated amyotrophic lateral sclerosis, where $\mathrm{NfH}$ may serve as a marker of prognosis ${ }^{523}$ and treatment response. ${ }^{524}$

TMEM106B rs1990622 polymorphisms). CSF progranulin has been studied less than blood with no clear cut-off defined for the presence of a GRN mutation at present, and a relatively weak correlation seen between CSF and blood (eg, r=0.54 in GRN mutation carriers and $\mathrm{r}=0.21$ in healthy controls in one study). ${ }^{10}$ In fact, although the concentration of progranulin in blood is about 20 times higher than that in CSF, most progranulin in CSF is brain-derived (with a calculated intrathecal fraction of $86 \%){ }^{11}$ Lastly, the majority of studies have used a small set of (non-fully validated) commercially available ELISAs to measure progranulin concentrations so far (eg, Adipogen, R+D, Biovendor), and while concentrations seem highly correlated between assays, ${ }^{12}$ absolute levels are different. Although the epitopes detected by the capture and detection antibodies are not known (or available) for these assays, the high correlation suggests that they may well measure similar forms of progranulin but that they have not been standardised to each other: a certified reference material and an external quality control programme for progranulin would solve this issue and would allow for standardisation of reference and decision limits across assays and laboratories. Nonetheless, these assays are already being used (with laboratory-specific reference and decision limits) not just clinically to detect the presence of mutations but also to detect treatment response in therapeutic trials; for example, in an early-phase trial of a monoclonal antibody against sortilin, plasma and CSF progranulin levels were reported to have been increased back into the normal range following treatment. ${ }^{13}$

\section{Dipeptide repeat proteins (DPRs)}

C9orf72-related FTD/ALS is caused by a GGGGCC hexanucleotide repeat expansion in the non-coding region of the gene. The pathogenic repeat size varies from probably more than around 30 to thousands, although the lower limit of pathogenicity remains undetermined. One of the key mechanisms of toxicity of large expansions is the production of DPRs by repeatassociated non-AUG (RAN) translation: C9orf72 RNA repeats can be translated through RAN translation producing five DPRs poly (GA), poly (GR), poly(PR), poly(PA) and poly(GP). A number of studies have tried to measure levels of these DPRs in CSF, and so far, only poly(GP) levels have been found to be measurable, with raised levels in both the presymptomatic and symptomatic periods (figure 2B) independent of clinical phenotype or stage of disease. ${ }^{14} 15$ Although not currently used clinically, it may become more widely used in a similar way to progranulin levels, allowing detection of an expansion prior to genetic screening (particularly if DPRs could be detected peripherally rather than in CSF). However, its more common use is likely to be as an important biomarker of treatment response in therapeutic trials, for example, in forthcoming antisense oligonucleotide therapy trials. So far, biomarker studies have used the Mesoscale Discovery platform (see box 2), with the current assay showing levels overlapping with controls in some C9orf72 expansion carriers-future studies using more sensitive techniques such as Simoa (box 2) may improve the dynamic range of the assay and separation of cases from controls, which will be very important for therapeutic trials aiming to show a decrease in level with treatment.

\section{Other genetic causes of FTD}

There are currently no specific biomarkers that can identify $M A P T$ mutations in biofluids nor any of the rarer genetic causes. TBK1 mutations, similarly to GRN, are usually frameshift, nonsense or splice site, and cause haploinsufficiency-it would therefore be theoretically possible to predict the presence of a pathogenic mutation through the detection of reduced TBK1 protein levels in blood or CSF, although no reliable methods are currently available.

\section{Sporadic FTD}

Determining the pathological nature of sporadic forms of FTD during life is much more difficult than the genetic forms. Around $50 \%$ of sporadic FTD is associated with a TDP-43 proteinopathy, $45 \%$ with a tauopathy and $5 \%$ with a FET proteinopathy, but specific biomarkers for the presence of these proteins do not currently exist.

\section{TDP-43}

TDP-43 proteinopathies are characterised by insoluble neuronal cytoplasmic or intranuclear inclusions and glial cytoplasmic inclusions, which aggregate in the cells. Cell death results in the release of TDP-43 and, therefore, its levels in CSF may increase. 'Total' TDP-43 levels have been measured in CSF with levels 

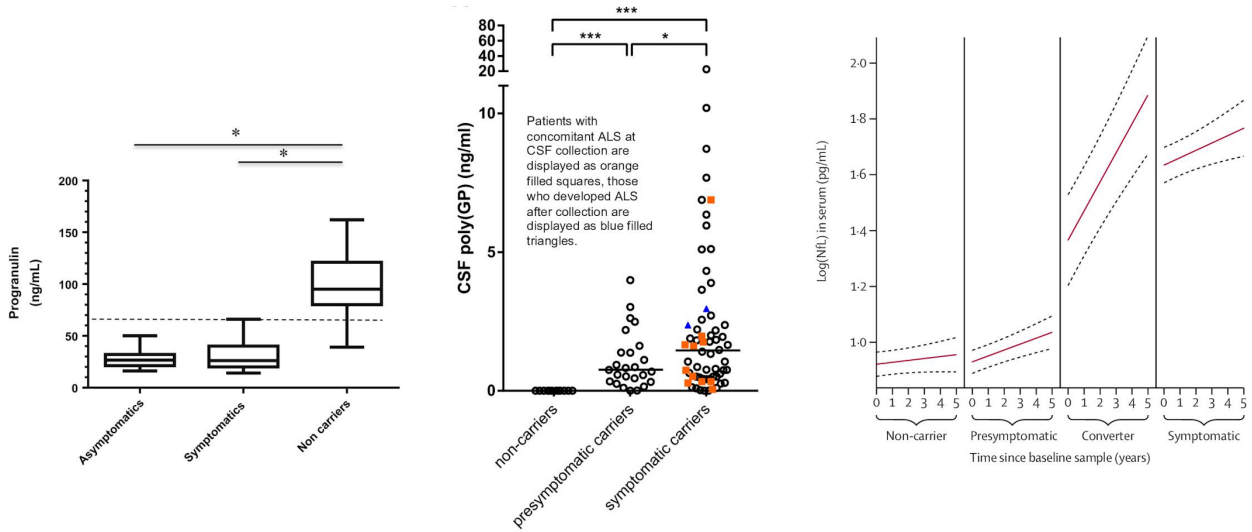

Figure 2 Key biomarkers in FTD: (A) plasma progranulin levels in GRN mutation carriers (from Galimberti et al ${ }^{549}$ ); (B) CSF poly(GP) DPR protein levels in C9orf72 mutation carriers (from Meeter et $a^{(550}$ ); (C) longitudinal serum NfL protein levels in genetic FTD (from van der Ende et a ${ }^{S 19}$ ). ALS, amyotrophic lateral sclerosis; C9orf72, chromosome 9 open reading frame 72; CSF, cerebrospinal fluid; DPR, dipeptide repeat; FTD, frontotemporal dementia; NfL, neurofilament light chain.

found to be higher than controls in some studies ${ }^{16-18}$ but with substantial overlap with controls and those with tauopathies, ${ }^{19}$ and therefore poor diagnostic accuracy for the presence of a TDP-43 proteinopathy. Similar findings of raised TDP-43 have been found in blood, ${ }^{20}$ with overall concentrations much higher than those seen in CSF. In fact, the total TDP-43 blood to CSF ratio is about 200:1, with one study suggesting that when found in CSF, this form of TDP-43 actually originates from the blood. ${ }^{21}$

Discovering a pathogenic (or pathology-associated) form of TDP-43 to measure in biofluids has led to studies of phosphorylated forms of TDP-43 (pTDP-43) and while this has been detected in plasma of patients at higher concentrations than controls, ${ }^{22} 23$ again there was substantial overlap with controls. No studies have managed to measure pTDP-43 in lumbar CSF so far.

Tau

Primary tauopathies include Pick's disease, progressive supranuclear palsy (PSP), corticobasal degeneration (CBD), globular glial tauopathy and MAPT mutations. So far, no specific tau biofluid measures of these disorders have been discovered. CSF concentrations of 'total' tau and phosphorylated forms of tau ( $\mathrm{p}$-tau181 and p-tau231) are variable in the different forms of FTD but, overall, are generally lower than in AD. Studies of tau protein fragments have so far not yielded any specific primary tauopathy markers. ${ }^{2425}$ More promising assays may arise from the real-time quaking-induced conversion method, which is currently under investigation for Pick's disease and the 4R-tauopathies. ${ }^{26-28}$

FET

These proteinopathies are rare causes of FTD, and as yet, no studies have shown their presence in CSF or blood.

\section{SPECIFIC MOLECULAR PATHWAYS IN FTD}

While some molecular processes occur across different forms of FTD, each pathogenetic form has unique features. Here we focus on studies of specific disease-associated proteins and unbiased proteomic investigations performed in specific forms of FTD.

\section{Progranulin}

Progranulin is a glycoprotein found mainly in neurons and microglia, and is involved in numerous cellular processes (figure 3), including neuroinflammation, lysosomal function and growth. It is a precursor protein, broken down into a number of smaller peptides, known as granulins 1-7 (previously known as A-G) and para-GRN. These proteins have key lysosomal and inflammatory roles and have been shown to promote TDP-43 accumulation and toxicity. However, due to their size and lack of specific antibodies, granulins have yet to be measured in biofluids. Nonetheless, they may prove to be important measures of treatment response in trials of GRN-targeted therapies; for example, unpublished work from one company show that their compound increases lysosomal granulins, while a sortilin-blocking monoclonal antibody decreases them in induced pluripotent stem cell-derived neurons. ${ }^{29}$ A mass spectrometric approach to the identification of specific progranulin breakdown fragments may be required.

Other proteins closely associated with progranulin include prosaposin, sortilin and secretory leucocyte protease inhibitor (SLPI). Prosaposin, similar to progranulin, performs different functions both intracellularly and extracellularly, including regulation of lysosomal enzymes and neuroprotection of glial cells. It is also a precursor protein, broken down into four saposins, which are involved in the breakdown of sphingolipids. Progranulin binds to prosaposin, and both proteins are trafficked into the lysosome, with studies showing that progranulin is important in mediating prosaposin and saposin levels in both neuronal and glial cells. No studies of prosaposin concentrations have yet been reported in FTD. Sortilin, a member of the Vps10p domain receptor family, is involved in the endocytosis of progranulin into the lysosome, forming a key receptor in progranulin functioning. Sortilin levels have been measured in the biofluids of ageing individuals, showing a strong positive correlation with progranulin levels in CSF but not in plasma, ${ }^{30}$ but have yet to be measured in those with GRN mutations. SLPI is an inhibitor of the serine protease, elastase, which is known to break down progranulin into the granulins. Levels have been investigated in one study, which showed significantly higher plasma concentrations in symptomatic GRN mutation carriers compared with both presymptomatic mutation carriers and controls. ${ }^{31}$ Interestingly, higher SLPI levels were associated with a later age of onset, with the authors suggesting that SLPI has a role in regulating penetrance. 


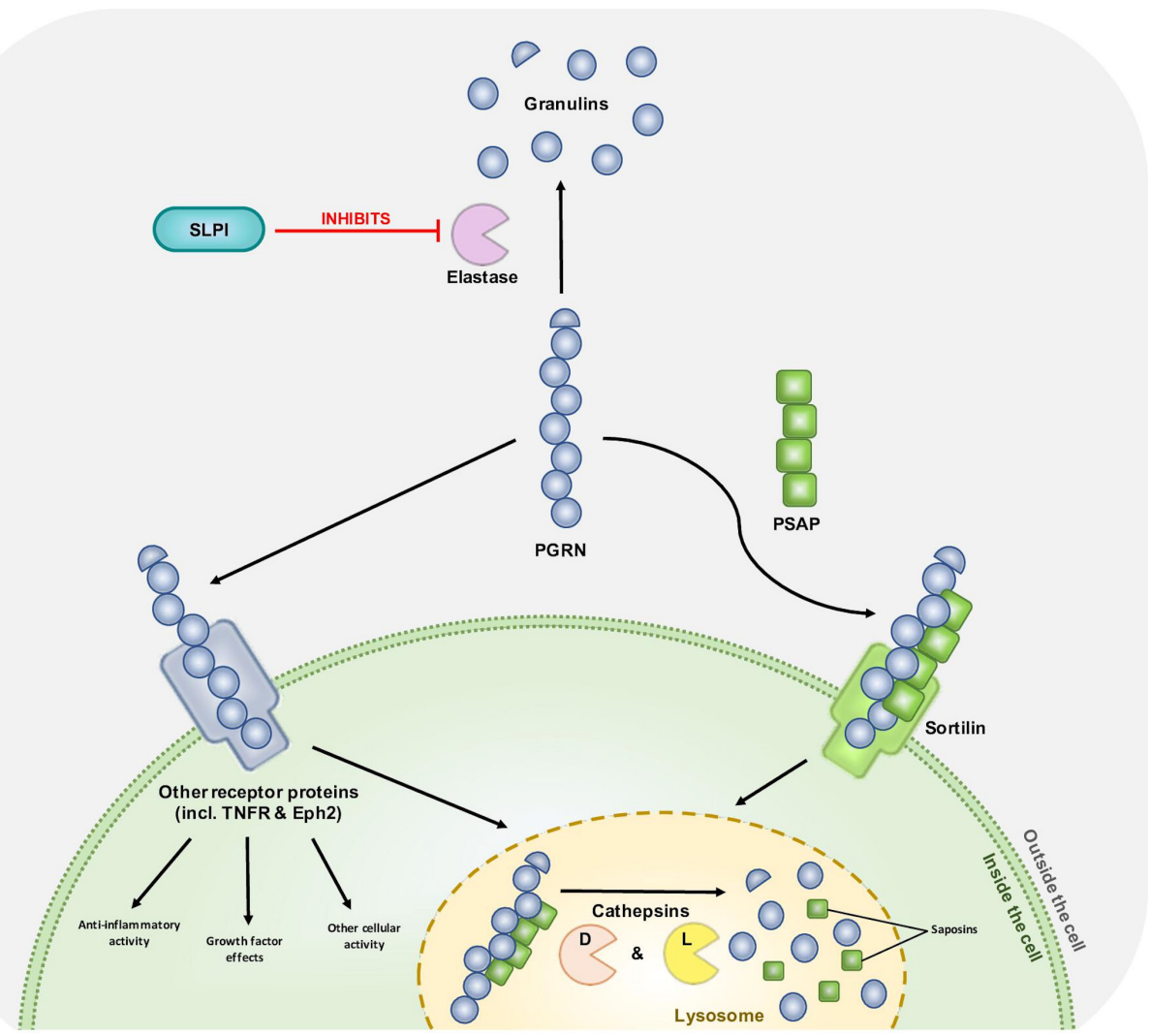

Figure 3 Biological pathways of progranulin. This diagram depicts the biological interactions of PGRN inside and outside the cell. Eph2, ephrin receptor 2; PGRN, progranulin; PSAP, prosaposin; SLPI, secretory leucocyte protease inhibitor; TNFR, tumour necrosis factor receptor.

An unbiased proteomic study using mass spectrometry in CSF samples of GRN mutation carriers showed significantly reduced levels of five proteins in symptomatic patients compared with controls: neuronal pentraxin receptor (NPTXR), receptortype tyrosine-protein phosphatase $\mathrm{N} 2$, neurosecretory protein VGF, chromogranin-A and V-set and transmembrane domaincontaining protein $2 \mathrm{~B} .{ }^{32}$ More work is needed to understand how specific these markers are to GRN mutation carriers (as many were also found to be abnormal in C9orf72 and MAPT mutation carriers as well) ${ }^{32}$ and what their role is in the GRN pathophysiological pathway.

\section{Chromosome 9 open reading frame 72}

Less is known about other interacting proteins within the C9orf72 pathways and few proteomic studies have been performed. However, a recent study compared the CSF proteome of C9orf72associated FTD and C9orf72-associated amyotrophic lateral sclerosis (ALS) and showed over 200 proteins were significantly different between the groups with neurofilament medium polypeptide, chitotriosidase and ubiquitin carboxyl-terminal hydrolase isozyme L1 among the proteins higher in the ALS group, and NPTXR decreased in the FTD group. ${ }^{33}$ As trials of C9orf72 mutation carriers may well include both people with ALS and FTD, it will be important to better understand which biomarkers are specific to C9orf72-related disease across the phenotypes, which are specific to one or other of C9orf72-associated FTD or C9orf72-associated ALS, and which are abnormal due to the presence of FTD or ALS independent of the fact the syndrome is due to a C9orf72 expansion. ${ }^{33}$ Such studies may also help in prediction of the subsequent phenotype in asymptomatic mutation carriers, although at present, proteomic investigations have found few changes before symptom onset.
NEUROINFLAMMATION, LYSOSOMAL FUNCTION AND SYNAPTIC HEALTH: MAJOR MOLECULAR MECHANISMS OF FTD

\section{Markers of neuroinflammation}

Neuroinflammation is a complex and multistage process involving activation of specific cells within the central nervous system (mainly microglia and astrocytes) and release of a series of pro- and anti-inflammatory factors. Recent evidence suggests that dysregulation of neuroinflammatory mechanisms may be involved in the pathophysiological process of FTD (reviewed in Bright $\left.e t a l^{34}\right)$. As expected from such a complex process, there are multiple molecules that can be measured, each highlighting dysfunction of specific parts of the inflammatory response (table 1). Here, we divide them into first, markers of glial cell activation; second, cytokines and chemokines; third, the complement system; and finally, the resolution pathway. Related to neuroinflammation, disruption of the blood-brain barrier (BBB), and angiogenesis may well also play a part in the pathophysiology of FTD and are reviewed in this section as well.

\section{Glial cell activation}

Glial cells, such as microglia and astrocytes, are pivotal to the inflammatory response in the brain. This glial activation leads to the production of inflammatory cytokines and chemokines, which induce changes in cell differentiation and morphology in response to stress. Different fluid biomarkers of glial activation have been measured, including soluble triggering receptor expressed on myeloid cells 2 (TREM2) and macrophage-derived chitinases, including chitotriosidase (CHIT1) and YKL-40 (otherwise known as chitinase-3-like protein 1 or CHI3L1). 
Table 1 Markers of inflammation in FTD

\begin{tabular}{|c|c|c|c|c|c|c|c|c|c|c|}
\hline \multirow[b]{2}{*}{ Fluid biomarkers } & \multirow[b]{2}{*}{ Biological function } & \multirow[b]{2}{*}{ Unspecified FTD } & \multirow[b]{2}{*}{ PSP } & \multicolumn{2}{|c|}{ FTD-GRN } & \multicolumn{2}{|c|}{ FTD-C9orf72 } & \multicolumn{2}{|c|}{ FTD-MAPT } & \multirow[b]{2}{*}{ Ref. } \\
\hline & & & & $\mathbf{P}$ & S & $\mathbf{P}$ & S & $\mathbf{P}$ & S & \\
\hline GFAP & Marker of astrogliosis & $\uparrow$ & & - & $\uparrow$ & - & - & - & - & 4041 \\
\hline STREM2 & Microglial activation marker & - & & & $\uparrow$ & & - & & - & 3536 \\
\hline YKL40 & Proinflammatory marker of activated astrocytes & $\uparrow$ & & & $\uparrow$ & & - & & $\uparrow$ & 3738 \\
\hline CHIT1 & Microglial activation marker & & & & $\uparrow$ & & - & & - & 3738 \\
\hline IL-1 $\beta$ & Cytokine with largely proinflammatory activity & & $\uparrow$ & & & & & & & 51 \\
\hline \multirow[t]{2}{*}{ IL-6 } & Cytokine with largely proinflammatory activity & $\uparrow$ & $\uparrow$ & - & $\uparrow$ & & - & & & 4344464751 \\
\hline & & - & & & - & & & & & \\
\hline IL-8 (CXCL8) & Cytokine with largely proinflammatory activity & $\uparrow$ & & & & & & & & 44 \\
\hline IL-11 & Cytokine with largely anti-inflammatory activity & $\uparrow$ & & & & & & & & 47 \\
\hline IL-12 & Cytokine with largely proinflammatory activity & $\downarrow$ & & & & & & & & 48 \\
\hline \multirow[t]{2}{*}{ IL-15 } & Cytokine with largely proinflammatory activity & $\uparrow$ & & & $\downarrow$ & & & & & 4249 \\
\hline & & & & & - & & & & & \\
\hline IL-18 & Cytokine with largely proinflammatory activity & - & & - & - & & & & & 46 \\
\hline \multirow[t]{3}{*}{ TNF- $\alpha$} & Cytokine with largely proinflammatory activity & $\uparrow$ & $\uparrow$ & - & $\uparrow$ & & & & & $4650-52$ \\
\hline & & & & & - & & & & & \\
\hline & & & & & $\downarrow$ & & & & & \\
\hline IP-10/CXCL10 & Chemokine with largely proinflammatory activity & - & & & $\uparrow$ & & & & & 4244 \\
\hline MCP-1/CCL2 & Chemokine with largely proinflammatory activity & $\uparrow$ & & & - & & & & & 4244 \\
\hline RANTES/ CCL5 & Cytokine with largely proinflammatory activity & $\downarrow$ & & & $\downarrow$ & & & & & 42 \\
\hline TGF- $\beta$ & Cytokine with largely anti-inflammatory activity & $\uparrow$ & & & & & & & & 50 \\
\hline
\end{tabular}

Blue, in cerebrospinal fluid; green, in blood and cerebrospinal fluid; red, in blood; yellow, in neuron-derived exosomes from plasma.

- indicates (unchanged levels); $\uparrow$ indicates increased levels; $\downarrow$ indicates decreased levels; all compared with controls.

CHIT1, chitotriosidase; C9orf72, chromosome 9 open reading frame 72; FTD, frontotemporal dementia; GFAP, glial fibrillary acidic protein; GRN, progranulin; IL, interleukin; MAPT, microtubule-

associated protein tau; MCP-1, monocyte chemoattractant protein-1; P, presymptomatic; PSP, progressive supranuclear palsy; RANTES, regulated on activation, normal T cell expressed and secreted; Ref, reference; S, symptomatic; sTREM2, soluble triggering receptor expressed on myeloid cells 2 ; TGF- $\beta$, transforming growth factor $\beta$; TNF- $\alpha$, tumour necrosis factor $\alpha$.

While TREM2 appears to be a marker of microglial activation in $\mathrm{AD}$, its status in FTD remains uncertain. Some studies have shown increases in an FTD cohort, ${ }^{35}$ while others have only shown increases when the cohort is stratified into specific pathogenetic forms. ${ }^{36}$

Similarly to TREM2, the chitinase proteins have been shown to be increased in the CSF of some cohorts but not others, ${ }^{33} 3738$ with some forms of FTD more likely to show increases than others (eg, those with associated ALS). Larger sample sizes in more well-defined cohorts are needed here, including examination within presymptomatic mutation carriers in genetic forms of FTD. Plasma levels of the chitinases have generally been found to be similar to controls in all groups investigated so far. ${ }^{39}$

Glial fibrillary acidic protein (GFAP), a marker of astrocytic activation/astrogliosis, has also been measured at increased levels in the CSF of people with FTD. ${ }^{39}{ }^{40}$ While levels in plasma were not elevated in an initial study, ${ }^{39}$ a more recent report in a large genetic cohort showed that GFAP was significantly increased in symptomatic GRN mutation carriers, ${ }^{41}$ with levels likely to be increasing just prior to symptom onset.

\section{Cytokines and chemokines}

Cytokines and chemokines are factors produced by glial cells in response to stressors, and quite a number have now been measured in different FTD biomarker studies (table 1).

CSF levels of a proinflammatory CC family cytokine, RANTES, are significantly reduced in both an unspecified FTD cohort and GRN mutation carriers compared with controls. ${ }^{42}$ Conversely, MCP-1, another proinflammatory cytokine of this family, is increased in the CSF of an FTD cohort overall and unchanged in GRN mutation carriers, suggesting complex underlying mechanisms. However, in both cases, this was not mirrored in serum, highlighting differences between the biological fluids and the need for further investigation. Similar paradoxical findings have been shown in other studies, for example, plasma levels of the proinflammatory interleukin (IL), IL-6, have been shown to be increased in FTD,${ }^{43}$ and yet CSF levels for the antiinflammatory interleukin, IL-8, are also increased. ${ }^{44}$ This may simply demonstrate the complexity of the inflammatory signature of FTD but could also indicate the importance of stratifying cohorts by pathology, clinical diagnosis, genetic group or disease stage. ${ }^{45-52}$ For example, one study which stratified their cohort by pathology, showed that CSF levels of proinflammatory interleukins, IL-17 and IL-23 were significantly reduced in those with TDP-43 pathology compared with those with tau pathology, whereas the tumour necrosis factor (TNF) family of cytokines, TRAILR3 and FasL, were significantly increased in those with TDP-43 pathology compared with those with tau pathology. ${ }^{45}$ Following this trend, a number of cytokines and chemokines are significantly different in GRN mutation carriers compared with non-GRN-FTD, including the interleukin IL-15, the CXC family cytokine IP-10 and TNF family cytokine, TNF- $\alpha{ }^{42}$

\section{Complement system}

There is some evidence to suggest a role for the complement system in the pathology of FTD, but only a small number of studies have so far examined complement proteins in biofluids. One study showed no difference in GRN mutation carriers compared with controls in either CSF C1qa and C3b, although both measures increased as cognition declined in the cohort, ${ }^{53}$ while another study showed raised C4d in patients with PSP. ${ }^{54}$

\section{Resolution pathway}

Resolution of inflammation is the process that takes place to return the tissue to a homeostatic condition. During resolution, there are specialised proresolving mediators (SPMs) that are synthesised in endothelial cells, macrophages and neutrophils 
and actively participate in the transition from a proinflammatory state to a homeostatic one. Only one study so far has explored alterations in SPMs in CSF and plasma so far, showing decreased levels of annexin 1 in the plasma of people with bvFTD compared with $\mathrm{AD}$ and controls. ${ }^{55}$

\section{Blood-brain barrier}

The BBB is also an important aspect of the body's response to stressors, and a number of neuropathological studies suggest that it can be affected in neurodegeneration. However, as the $\mathrm{BBB}$ is a physical barrier a real neurochemical marker for this does not exist. Often the albumin CSF:blood ratio is claimed to be a marker for the BBB, but the albumin quotient more likely represents the CSF flow and is therefore perhaps better thought of as a measure of the CSF-blood barrier. If this flow is affected, higher levels of albumin will be present in the CSF, giving an increased albumin CSF:serum ratio. In FTD, the levels in an unspecified cohort are variable without any significant difference from controls, ${ }^{56}$ but studies of better phenotyped groups are needed.

\section{Angiogenesis}

Although not part of the inflammation pathway specifically, angiogenesis, that is the formation of new blood vessels, can occur with impaired BBB function and is stimulated by activated immune cells. Vascular endothelial growth factor (VEGF) levels are increased in the CSF of people with $\mathrm{FTD},{ }^{57}$ as are levels of another member of the VEGF family, placental growth factor, ${ }^{58}$ as well as angiogenin. ${ }^{59}$ Platelet-derived growth factor subunit $\mathrm{B}$ has been shown to be increased in the serum of a PSP/CBS group compared with controls, ${ }^{60}$ but other angiogenic factors have not been well studied. However, initial investigations of matrix metalloproteinases (MMPs), involved in angiogenesis as well as other processes (MMP-2 and MMP-9), show alterations in an FTD cohort. ${ }^{61}$

\section{Markers of lysosomal function}

The function of the lysosome in a cell is to break down and recycle proteins, ensuring cellular homeostasis and survival. Abnormal lysosomal function has been described in FTD in cellular models, but few markers of this have been measured so far in biofluids (table 2). One study has shown that lysosomalassociated membrane protein 1 (LAMP1) levels are significantly higher in plasma exosomes from patients with AD but not FTD. ${ }^{62}$ However, limited sample sizes restricted the further stratification of the FTD group, which is likely to be an important factor; for example, in a study of CSF from people with pathologically confirmed PSP and CBD, LAMP1 and LAMP2 were increased in
CBD, while another marker lysozyme was increased in both PSP and CBD compared with controls. ${ }^{63}$

A group of cysteine proteases, known as cathepsins, may also form promising markers of lysosomal function in FTD; for example, cathepsin D levels measured in plasma exosomes are significantly higher in FTD and AD compared with controls. ${ }^{62}$ However, research into these enzymes is relatively limited so far.

Lastly, and as also discussed previously, a number of the proteins involved in the pathophysiology of GRN-related FTD are key to lysosomal function, including prosaposin (and the saposins), as well as glucocerebrosidase. Other lysosomal enzymes have been poorly studied in FTD, although one study showed lower levels of alpha-mannosidase in CSF compared with healthy controls. ${ }^{64}$

\section{Markers of synaptic and neurotransmitter function}

Progressive synaptic dysfunction and loss have been shown to occur in FTD, and therefore, it is reasonable to expect that any changes in synaptic proteins in brain tissue may be reflected in changes within the CSF (or potentially the blood) of people with FTD (table 3).

\section{Presynaptic markers}

One study recently compared the levels of the synaptic proteins synaptosomal-associated protein, $25 \mathrm{kDa}$ (SNAP-25) and synaptotagmin in CSF samples from people with AD and FTD and age-matched controls. ${ }^{65}$ SNAP-25 binds to synaptobrevin in the presynaptic plasma membrane to form the soluble NSF attachment protein receptor (SNARE) complex, involved in vesicle fusion and release of neurotransmitters, while synaptotagmin is a vesicle protein that acts as a $\mathrm{Ca}^{2+}$ sensor triggering vesicle fusion on calcium influx. None of the synaptic proteins showed altered levels in FTD patients when compared with controls, but both of them were increased in an $\mathrm{AD}$ cohort. ${ }^{65}$ This is in contrast to a previous study that measured exosome-derived proteins in plasma within a group of people with FTD and showed decreased levels of synaptotagmin, as well as synaptophysin, the most abundant integral synaptic vesicle protein that regulates SNARE assembly and vesicle fusion. ${ }^{66}$ In this same study, GAP-43, another presynaptic protein that interacts with the SNARE complex and plays a role in $\mathrm{Ca}^{2+}$-dependent vesicle fusion, was not altered in FTD, whereas the peripheral vesicle protein synapsin 1 , involved in vesicle trafficking, was increased. ${ }^{66}$

While beta-synuclein, a further candidate presynaptic marker, has only shown elevated levels in the CSF of those with $\mathrm{AD}$ and not FTD, ${ }^{67}$ other potential markers for FTD include Rab3A, a protein essential in intracellular transport and in sustaining a reserve of vesicles ready for release, which is decreased in the tissue of people with FTD and shows promise as a CSF marker

Table 2 Lysosomal markers

\begin{tabular}{|c|c|c|c|c|c|c|c|c|c|c|}
\hline \multirow[b]{2}{*}{ Fluid biomarkers } & \multirow[b]{2}{*}{ Biological function } & \multirow[b]{2}{*}{ Unspecified FTD } & \multirow[b]{2}{*}{ PSP } & \multicolumn{2}{|c|}{ FTD-GRN } & \multicolumn{2}{|c|}{ FTD-C9orf72 } & \multicolumn{2}{|c|}{ FTD-MAPT } & \multirow[b]{2}{*}{ Ref. } \\
\hline & & & & $\mathbf{P}$ & S & $\mathbf{P}$ & S & $\mathbf{P}$ & s & \\
\hline LAMP1 & Glycoprotein involved in regulation of lysosomal function & - & - & & & & & & & 6263 \\
\hline Cathepsin D & $\begin{array}{l}\text { Protease involved in degrading proteins such as progranulin in the } \\
\text { lysosome }\end{array}$ & $\uparrow$ & & & & & & & & 62 \\
\hline$\alpha$-Mannosidase & Lysosomal hydrolase & $\downarrow$ & & & & & & & & 64 \\
\hline Lysozyme & Innate immunity enzyme & & $\uparrow$ & & & & & & & 63 \\
\hline
\end{tabular}

Blue, in cerebrospinal fluid; green, in blood and cerebrospinal fluid; red, in blood; yellow, in neuron-derived exosomes from plasma.

- indicates (unchanged levels); $\uparrow$ indicates increased levels; $\downarrow$ indicates decreased levels; all compared with controls.

C9orf72, chromosome 9 open reading frame 72; FTD, frontotemporal dementia; GRN, progranulin; LAMP1, lysosomal-associated membrane protein 1; MAPT, microtubule-

associated protein tau; P, presymptomatic; PSP, progressive supranuclear palsy; Ref, reference; S, symptomatic. 
Table 3 Markers of synaptic and neurotransmitter function

\begin{tabular}{|c|c|c|c|c|c|c|c|c|c|c|}
\hline \multirow[b]{2}{*}{ Fluid biomarkers } & \multirow[b]{2}{*}{ Biological function } & \multirow{2}{*}{$\begin{array}{l}\text { Unspecified } \\
\text { FTD }\end{array}$} & \multirow[b]{2}{*}{ PSP } & \multicolumn{2}{|c|}{ GRN } & \multicolumn{2}{|c|}{ C9orf72 } & \multicolumn{2}{|c|}{ MAPT } & \multirow[b]{2}{*}{ Ref. } \\
\hline & & & & $\mathbf{P}$ & s & $\mathbf{P}$ & s & $\mathbf{P}$ & $\mathrm{s}$ & \\
\hline SNAP-25 & Vesicle fusion and release of neurotransmitters & - & & & & & & & & 65 \\
\hline \multirow[t]{2}{*}{ Synaptotagmin } & \multirow{2}{*}{$\mathrm{Ca}^{2+}$ sensor triggering vesicle fusion on calcium influx } & - & & & & & & & & \multirow[t]{2}{*}{6566} \\
\hline & & $\downarrow$ & & & & & & & & \\
\hline Synaptophysin & Synaptic vesicle protein that regulates SNARE assembly and vesicle fusion & $\downarrow$ & & & & & & & & 66 \\
\hline GAP-43 & $\mathrm{Ca}^{2+}$-dependent vesicle fusion & - & & & & & & & & 66 \\
\hline Synapsin 1 & Vesicle trafficking & $\uparrow$ & & & & & & & & 66 \\
\hline \multirow[t]{2}{*}{ Neurogranin } & \multirow{2}{*}{$\begin{array}{l}\text { Long-term potentiation and synaptic plasticity and regulates intracellular } \mathrm{Ca}^{2+} \\
\text { concentration }\end{array}$} & - & & & & & & & & \multirow[t]{2}{*}{6566} \\
\hline & & $\downarrow$ & & & & & & & & \\
\hline Synaptopodin & Regulates intracellular $\mathrm{Ca}^{2+}$ concentration & $\downarrow$ & & & & & & & & 66 \\
\hline Reelin & $\begin{array}{l}\text { Regulation of filopodia formation, dendrite growth and spine formation, and } \\
\text { synaptogenesis, modulation of synaptic plasticity and neurotransmitter release }\end{array}$ & $\uparrow$ & & & & & & & & 68 \\
\hline NPTXR & Organisation of excitatory and inhibitory synapses & $\downarrow$ & & - & $\downarrow$ & - & $\downarrow$ & - & - & 69 \\
\hline NPTX2 & Modulation of strength of excitatory synapses & & & - & $\downarrow$ & - & $\downarrow$ & - & - & 69 \\
\hline DA & Neurotransmitter & $\uparrow$ & & & & & & & & 71 \\
\hline DOPAC & DA metabolite & $\downarrow$ & & & & & & & & 71 \\
\hline
\end{tabular}

Blue, in cerebrospinal fluid; green, in blood and cerebrospinal fluid; red, in blood; yellow, in neuron-derived exosomes from plasma.

- indicates (unchanged levels); $\uparrow$ indicates increased levels; $\downarrow$ indicates decreased levels; all compared with controls.

C9orf72, chromosome 9 open reading frame 72; DA, dopamine; DOPAC, 3,4-dihydroxyphenylacetic acid; FTD, frontotemporal dementia; GAP-43, growth associated protein, 43 kDa; GRN, progranulin; MAPT, microtubule-associated protein tau; NPTX2, neuronal pentraxin-2; NPTXR, neuronal pentraxin receptor; P, presymptomatic; PSP, progressive supranuclear palsy; Ref, reference; S, symptomatic; SNAP-25, synaptosomal-associated protein, $25 \mathrm{kDa}$; SNARE, soluble NSF attachment protein receptor.

of synapse loss in other disorders, and synaptic vesicle glycoprotein $2 \mathrm{~A}$, currently under investigation to measure synaptic density with positron emission tomography (PET) imaging but not yet measured in CSF.

\section{Postsynaptic markers}

Neurogranin is involved in long-term potentiation and synaptic plasticity and regulates intracellular $\mathrm{Ca}^{2+}$ concentration. Increased concentrations of neurogranin in CSF in $\mathrm{AD}$ predict cognitive decline from $\mathrm{MCI}$ to $\mathrm{AD}$. In FTD, levels of neurogranin in CSF seem to remain unchanged when compared with controls. ${ }^{65}$ However, when measured in exosomes in plasma, neurogranin was significantly decreased when compared with controls in FTD ${ }^{66}$ Synaptopodin regulates intracellular $\mathrm{Ca}^{2+}$ concentration, and decreased levels were also shown in the same study. ${ }^{66}$

\section{Other synaptic markers}

Reelin is an extracellular matrix glycoprotein with roles including regulation of filopodia formation, dendrite growth and spine formation and synaptogenesis, as well as modulation of synaptic plasticity and neurotransmitter release. One small study has previously shown increased levels in FTD in CSF compared with controls. $^{68}$

Recent studies have investigated the neuronal pentraxin family of proteins as synaptic dysfunction markers. ${ }^{3369}$ Neuronal pentraxins are multifunctional proteins implicated in synaptic plasticity. Decreased CSF levels of neuronal pentraxin receptor (NPTXR) have been shown in symptomatic genetic FTD, while neuronal pentraxin-2 (NPTX2) is decreased in symptomatic GRN and C9orf72 mutation carriers but not in MAPT mutation carriers. Levels of NPTX2 in CSF correlate with disease progression as well as NfL levels and can predict symptom onset. ${ }^{69}$

\section{Neurotransmitter release}

Serotonergic, dopaminergic, glutamatergic and GABAergic pathways have all been shown to be altered in FTD. One study has reported that increased activity of dopaminergic neurotransmission and altered serotonergic modulation of dopaminergic neurotransmission is associated with agitated and aggressive behaviour, whereas degeneration of the noradrenergic neurotransmitter system might contribute to the cognitive deficits in FTD. ${ }^{70}$

An increase in dopamine (DA) and a decrease in its metabolite 3,4-dihydroxyphenylacetic acid (DOPAC) have been reported in CSF and blood in people with FTD and ALS when compared with controls, and the ratio of DOPAC to DA is able to discriminate FTD from ALS. ${ }^{71}$

\section{CIRCULATING NUCLEIC ACIDS}

While most DNA and RNA are found within cells, some nucleic acids are found circulating within the blood as well as other body fluids, including CSF. While cell-free DNA studies are under way in FTD, little has been reported about their role so far. However, more studies have focused on microRNAs (miRNAs), small non-coding RNA molecules involved in the regulation of gene expression. At present, most studies have investigated whether single miRNAs or combinations of multiple miRNAs in blood or CSF are able to differentiate FTD syndromes from controls or other neurodegenerative disorders ${ }^{72}{ }^{73}$; for example, in one study, a combination of three miRNAs (miR-663a, miR-502-3p and miR-206) classified FTD from controls with an accuracy of $84.4 \%{ }^{72}$ miRNAs may also act as staging markers; for example, one study showed a difference in two miRNAs (miR-204-5p and miR-632) in CSF between symptomatic and presymptomatic mutation carriers in genetic FTD. ${ }^{74}$

\section{FUTURE: CLINICAL TRIALS AND TREATMENT RESPONSE}

The future of FTD is an increasing number of therapeutic studies and potentially a point in time where most diagnosed patients are entering into clinical trials. It is important to remember that while many of these will be disease-modifying, trials of symptomatic treatments will also continue to be important e.g. improvement in altered eating behaviour (see box 4 ). Discovery of better markers of staging (box 5) and therapeutic response, in particular, will be required, and while new technologies (box 2) that allow multiple proteins to be measured at the same time in increasingly sensitive ways will be important, it will also be 
Box 4 Measures of hypothalamic function

Excessive eating and dietary changes are well recognised in frontotemporal dementia (FTD) and can be used to differentiate behavioural frontotemporal dementia (bvFTD) from Alzheimer's disease (AD). ${ }^{525-527}$ Studies investigating eating behaviour have shown altered metabolism, ${ }^{527-530}$ and so studies have begun to focus on molecular measures of appetite, and therefore also the hypothalamus, as it acts as a key control centre in modulating appetite through various highly interconnected nuclei that communicate through neuropeptides. ${ }^{\text {S26,531-534 }}$ Lower levels of ghrelin and cortisol, but higher levels of insulin, have been observed in bvFTD compared with controls and AD. ${ }^{S 35}$ In the same study, higher levels of leptin were found in those with marked hyperphagia. ${ }^{\mathrm{S} 5}$ These metabolic patterns are consistent with a state of satiety, which suggests the eating disturbances observed in FTD result from loss of inhibitory signals; however, the compensatory hormonal responses cannot reverse these symptoms. The first study investigating cerebrospinal fluid (CSF) agouti-related peptide (AgRP) in FTD found a sevenfold increase in people with TDP-43 pathology compared with people with tau pathology. ${ }^{45}$ A further study detected increased levels of AgRP in bvFTD and semantic variant PPA compared with controls, 527 also showing that both AgRP and leptin were predictors of body mass index. ${ }^{527}$ These findings of elevated AgRP, which stimulates appetite, suggest that neuropeptides may directly be involved in modulating eating behaviour in FTD and promote excessive eating. In a further study, decreased neuropeptide $Y$ levels were found in people with FTD compared with controls and patients with amyotrophic lateral sclerosis (ALS). ${ }^{\mathrm{S} 6}$ They also showed increased levels of leptin and insulin resistance in people with bvFTD and $\mathrm{ALS}_{1}{ }^{\mathrm{S36}}$ further complicating the picture of metabolic changes underlying eating disturbances in FTD. Few metabolic studies have focused on specific forms of FTD so far. However, in one study focused on GRN-related FTD, serum C-peptide, resistin and ghrelin were all increased. ${ }^{\mathrm{S37}}$ Lastly, hypothalamic proteins have been studied in relation to sleep in FTD, with CSF orexin levels being correlated to daytime somnolence ${ }^{\mathrm{S38}}$ in one study, and plasma orexin being lower in people with FTD compared with controls in another. ${ }^{\text {S39 }}$

Box 5 Staging of frontotemporal dementia (FTD) and proximity markers

Unlike in Alzheimer's disease, few markers of disease stage have been identified in FTD so far. 'Preclinical' FTD may be defined by the onset of detectable pathological changes, but at present, we have no biomarkers of tau, TDP-43 or FET that might theoretically become abnormal quite a number of years prior to symptom onset. While we do have markers of dipeptide repeat proteins (DPRs) in chromosome 9 open reading frame 72 expansion-related disease, further studies of when these become abnormal are required (as it is likely that in this group DPR pathology precedes TDP-43 pathology ${ }^{540}$ ). 'Prodromal' FTD may be defined by the onset of mild symptoms that do not meet diagnostic criteria, that is, mild behavioural, cognitive (and/or motor) impairment. During or just prior to this stage, we may define 'proximity measures', that is, those identifying a period prior to phenoconversion, and for FTD both neurofilament light chain, ${ }^{513,519}$ and glial fibrillary acidic protein in GRN mutation carriers $^{41}$ are candidates for these.

\section{Box 6 Frontotemporal dementia (FTD) cohorts}

As FTD is a rare disease with multiple phenotypes and pathogenetic causes, a substantial number of prior biomarker studies have involved relatively small sample sizes, reducing the power and likelihood of finding abnormalities. In recent years, however, larger, deeply phenotyped cohorts have been developed that have facilitated biomarker studies of increased size. In genetic FTD, these include the Genetic FTD Initiative (www. genfi.org) $)^{S 41}$ in Europe and Eastern Canada, and the ARTFL/ LEFFTDS (now ALLFTD: https://www.allftd.org) cohort in the USA and Western Canada, ${ }^{542,543}$ which collaborate through the FTD Prevention Initiative (www.thefpi.org). ${ }^{544}$ The ALLFTD cohort also includes sporadic FTD, and while there are no large multinational cohorts in Europe, large studies of sporadic FTD with biomarker analyses include the German FTLD consortium ${ }^{545}$ and DZNE FTD DESCRIBE cohort. ${ }^{S 46}$ Specific atypical parkinsonian cohorts include the UK PROSPECT study ${ }^{547}$ and the US 4RTNI cohort. ${ }^{548}$

imperative to look beyond the fluid biomarker field to consider multimodal combinations of measures that cross biofluids, MRI and PET, making use of new data science methods, including machine and deep learning. It is likely that such studies will take place within the context of large cohort studies (see box 6), avoiding the problems of small sample sizes that are inherent to so many of the published papers so far. Such studies will also pave the way for larger omics studies which have so far been relatively small and focused on proteomics despite initial evidence for abnormalities in metabolomics ${ }^{75-77}$ and lipidomics ${ }^{78-80}$ in FTD as well.

\section{CONCLUSION}

The past of FTD biomarkers as described here is a wide range of different markers, some more promising than others, but many examined in small single centre cohorts. The present is the recent introduction of more sensitive blood-based biomarker methods and the availability of larger sample collections from the well-characterised multinational genetic FTD cohorts, with great promise for markers such as NfL and GFAP. The future is trials and within this, the validation of multiplex biomarker panels targeted at specific pathogenetic forms of FTD, and eventually to individuals, providing a more personalised approach to outcome measures in upcoming trials.

\footnotetext{
Author affiliations

'UK Dementia Research Institute at University College London, UCL Queen Square Institute of Neurology, London, UK

${ }^{2}$ Dementia Research Centre, Department of Neurodegenerative Disease, UCL Queen Square Institute of Neurology, London, UK

${ }^{D}$ epartment of Neurodegenerative Diseases, Hertie Institute for Clinical Brain Research and Center of Neurology, University of Tübingen, Tübingen, Germany ${ }^{4}$ German Center for Neurodegenerative Diseases (DZNE), Tübingen, Germany ${ }^{5}$ Department of Neurology, University of Ulm, Ulm, Germany

${ }^{6}$ Division for Neurogeriatrics, Center for Alzheimer Research, Department of NVS, Karolinska Institutet, Stockholm, Sweden

${ }^{7}$ Unit for Hereditary Dementias, Theme Aging, Karolinska University Hospital, Solna, Sweden

${ }^{8}$ Department of Biomedical, Surgical and Dental Sciences, University of Milan, Centro Dino Ferrari, Milan, Italy

${ }^{9}$ Fondazione IRCCS Ca' Granda, Ospedale Maggiore Policlinico, Milan, Italy ${ }^{10}$ Department of Neurology, Erasmus Medical Centre, Rotterdam, The Netherlands ${ }^{11}$ Department of Psychiatry and Neurochemistry, Sahlgrenska Academy at the University of Gothenburg, Mölndal, Sweden
}

Acknowledgements The Dementia Research Centre is supported by Alzheimer's Research UK, Alzheimer's Society, Brain Research UK, and The Wolfson Foundation. 
This work was supported by the NIHR UCL/H Biomedical Research Centre, the Leonard Wolfson Experimental Neurology Centre (LWENC) Clinical Research Facility and the UK Dementia Research Institute, which receives its funding from UK DRI Ltd, funded by the UK Medical Research Council, Alzheimer's Society and Alzheimer's Research UK. MO has received funding from BMBF (FTLDc, PreFrontALS, Genfi-Prox), EU (Miriade, Fairpark II, Moodmarker), Thierry Latran Foundation and the ALS Association. CG is supported by grants from Schörling Foundation, Swedish FTD Initiative, Swedish Research Council (2015-02926, 2018-02754 and 2019-02248: JPND GENFI-PROX), Swedish Alzheimer's Foundation, Brain Foundation, Demensfonden, Stiftelsen för Gamla Tjänarinnor, Stohnes Foundation, and grants provided by Region Stockholm (ALF project). DG has received support from the EU Joint Programme, Neurodegenerative Disease Research (JPND) and the Italian Ministry of Health (PreFrontALS, grant 733051 042). JCVS was supported by the Dioraphte Foundation (grant 09-02-03-00), the Association for Frontemporal Dementias Research Grant 2009, The Netherlands Organisation for Scientific Research (NWO) (grant HCMI 056-13-018), ZonMw Memorabel (Deltaplan Dementie, project number 733051 042), Alzheimer Nederland and the Bluefield project. HZ is a Wallenberg Scholar supported by grants from the Swedish Research Council (\#2018-02532), the European Research Council (\#681712), Swedish State Support for Clinical Research (\#ALFGBG-720931), the Alzheimer Drug Discovery Foundation (ADDF), USA (\#2 01 809-2016862), and the UK Dementia Research Institute at UCL. Several authors of this publication are members of the European Reference Network for Rare Neurological Diseases (Project ID No 739 510). The JPND GENFI-PROX grant (2019-02248) provides support to JDR (through the MRC), MS and MO (through the DLR/BMBF), CG and JCVS.

Contributors IS, AS-E, CH and JDR performed the initial literature search and wrote the initial draft of the manuscript. MS, MO, CG, DG, ET, AJH, ELvdE, JCVS and $\mathrm{HZ}$ all critically reviewed the initial draft of the manuscript and contributed additional literature to the review. IS, AS-E, CH, MS, MO, CG, DG, ET, AJH, ELvdE, JCVS, HZ and JDR all contributed to the writing of the final version of the manuscript.

Funding JDR has received funding from an MRC Clinician Scientist Fellowship (MR/ M008525/1), the NIHR Rare Disease Translational Research Collaboration (BRC149/ NS/MH), the MRC UK GENFI grant (MR/M023664/1), and the Bluefield Project.

Competing interests $\mathrm{HZ}$ has served at scientific advisory boards for Denali, Roche Diagnostics, Wave Life Sciences, Samumed, Siemens Healthineers, Pinteon Therapeutics and CogRx; has given lectures in symposia sponsored by Fujirebio, Alzecure and Biogen; and is a cofounder of Brain Biomarker Solutions in Gothenburg $A B$, which is a part of the GU Ventures Incubator Program. JDR has served on medical advisory boards for lonis, Wave Life Sciences, Alector and Prevail.

Patient consent for publication Not required.

Provenance and peer review Commissioned; externally peer reviewed.

\section{ORCID iDs}

Imogen Joanna Swift http://orcid.org/0000-0002-9097-0152

Carolin Heller http://orcid.org/0000-0002-1934-6162

Markus Otto http://orcid.org/0000-0002-6647-5944

Daniela Galimberti http://orcid.org/0000-0002-9284-5953

Emma Louise van der Ende http://orcid.org/0000-0003-2570-8796

John Cornelis Van Swieten http://orcid.org/0000-0001-6278-6844

Jonathan Daniel Rohrer http://orcid.org/0000-0002-6155-8417

\section{REFERENCES}

1 Seelaar H, Rohrer JD, Pijnenburg YAL, et al. Clinical, genetic and pathological heterogeneity of frontotemporal dementia: a review. J Neurol Neurosurg Psychiatry 2011:82:476-86.

2 Rohrer JD, Guerreiro R, Vandrovcova J, et al. The heritability and genetics of frontotemporal lobar degeneration. Neurology 2009:73:1451-6.

3 Paterson RW, Slattery CF, Poole T, et al. Cerebrospinal fluid in the differential diagnosis of Alzheimer's disease: Clinical utility of an extended panel of biomarkers in a specialist cognitive clinic. Alzheimer's Res Ther 2018;10.

4 Thijssen EH, La Joie R, Wolf A, et al. Diagnostic value of plasma phosphorylated tau181 in Alzheimer's disease and frontotemporal lobar degeneration. Nat Med 2020;26:387-97.

5 Palmqvist S, Janelidze S, Quiroz YT, et al. Discriminative accuracy of plasma phospho-tau217 for Alzheimer disease vs other neurodegenerative disorders. JAMA 2020;324:772.

6 Gabelle A, Roche S, Gény C, et al. Decreased SA $\mathrm{PPP} \beta, A \beta 38$, and a $\beta 40$ cerebrospinal fluid levels in frontotemporal dementia. J Alzheimers Dis 2011;26:553-63.

7 Al Shweiki MR, Steinacker P, Oeckl P, et al. Neurofilament light chain as a blood biomarker to differentiate psychiatric disorders from behavioural variant frontotemporal dementia. J Psychiatr Res 2019;113:137-40.

8 Ghidoni R, Stoppani E, Rossi G, et al. Optimal plasma progranulin cutoff value for predicting null progranulin mutations in neurodegenerative diseases: a multicenter Italian study. Neurodegener Dis 2012;9:121-7.
9 Sellami L, Rucheton B, Ben Younes I, et al. Plasma progranulin levels for frontotemporal dementia in clinical practice: a 10-year French experience. Neurobiol Aging 2020;91:167.e1-167.e9.

10 Meeter LHH, Patzke H, Loewen G, et al. Progranulin levels in plasma and cerebrospinal fluid in granulin mutation carriers. Dement Geriatr Cogn Dis Extra 2016;6:330-40.

11 Feneberg E, Steinacker P, Volk AE, et al. Progranulin as a candidate biomarker for therapeutic trial in patients with ALS and FTLD. J Neural Transm 2016;123:289-96.

12 Willemse EAJ, Durieux-Lu S, van der Flier WM, et al. Stability of progranulin under pre-analytical conditions in serum and cerebrospinal fluid. J Alzheimers Dis 2016;53:107-16

13 BioSpace. Alector announces data from on-going phase $1 \mathrm{~B}$ trial demonstrating that AL001 reverses progranulin deficiency in frontotemporal dementia patients. Available: https://www.biospace.com/article/-alector-announces-data-from-on-going-phase-1btrial-demonstrating-that-al001-reverses-progranulin-deficiency-in-frontotemporaldementia-patients/ [Accessed 21 Jul 2020].

14 Gendron TF, Chew J, Stankowski JN, et al. Poly(GP) proteins are a useful pharmacodynamic marker for C9ORF72 -associated amyotrophic lateral sclerosis. Sci Trans/ Med 2017;9. doi:10.1126/scitransImed.aai7866. [Epub ahead of print: 29 Mar 2017].

15 Lehmer C, Oeckl P, Weishaupt JH, et al. Poly-GP in cerebrospinal fluid links C9orf72associated dipeptide repeat expression to the asymptomatic phase of ALS/FTD. EMBO Mol Med 2017;9:859-68.

16 Steinacker P, Hendrich C, Sperfeld AD, et al. TDP-43 in cerebrospinal fluid of patients with frontotemporal lobar degeneration and amyotrophic lateral sclerosis. Arch Neurol 2008;65:1481-7.

17 Kasai T, Kojima Y, Ohmichi T, et al. Combined use of CSF NFL and CSF TDP-43 improves diagnostic performance in ALS. Ann Clin Transl Neurol 2019;6:2489-502.

18 Noto Y-I, Shibuya K, Sato Y, et al. Elevated CSF TDP-43 levels in amyotrophic lateral sclerosis: specificity, sensitivity, and a possible prognostic value. Amyotroph Lateral Scler 2011;12:140-3.

19 Kuiperij HB, Versleijen AAM, Beenes M, et al. Tau rather than TDP-43 proteins are potential cerebrospinal fluid biomarkers for frontotemporal lobar degeneration subtypes: a pilot study. J Alzheimers Dis 2017;55:585-95

20 Foulds P, McAuley E, Gibbons L, et al. TDP-43 protein in plasma may index TDP-43 brain pathology in Alzheimer's disease and frontotemporal lobar degeneration. Acta Neuropathol 2008;116:141-6.

21 Feneberg E, Gray E, Ansorge 0, et al. Towards a TDP-43-based biomarker for ALS and FTLD. Mol Neurobiol 2018;55:7789-801.

22 Foulds PG, Davidson Y, Mishra M, et al. Plasma phosphorylated-TDP-43 protein levels correlate with brain pathology in frontotemporal lobar degeneration. Acta Neuropathol 2009;118:647-58.

23 Suárez-Calvet M, Dols-Icardo 0, Lladó A, et al. Plasma phosphorylated TDP-43 levels are elevated in patients with frontotemporal dementia carrying a C9orf72 repeat expansion or a GRN mutation. J Neurol Neurosurg Psychiatry 2014;85:684-91.

24 Foiani MS, Cicognola C, Ermann N, et al. Searching for novel cerebrospinal fluid biomarkers of tau pathology in frontotemporal dementia: an elusive quest. J Neurol Neurosurg Psychiatry 2019;90:740-6.

25 Barthélemy NR, Gabelle A, Hirtz C, et al. Differential mass spectrometry profiles of tau protein in the cerebrospinal fluid of patients with Alzheimer's disease, progressive supranuclear palsy, and dementia with Lewy bodies. J Alzheimers Dis 2016;51:1033-43.

26 Saijo E, Ghetti B, Zanusso G, et al. Ultrasensitive and selective detection of 3-repeat tau seeding activity in Pick disease brain and cerebrospinal fluid. Acta Neuropathol 2017;133:751-65.

27 Saijo E, Metrick MA, Koga S, et al. 4-Repeat tau seeds and templating subtypes as brain and CSF biomarkers of frontotemporal lobar degeneration. Acta Neuropathol 2020;139:63-77

28 Metrick MA, Ferreira NdoC, Saijo E, et al. A single ultrasensitive assay for detection and discrimination of tau aggregates of Alzheimer and Pick diseases. Acta Neuropathol Commun 2020;8:22.

29 Arkuda Therapeutics. Discovery of small molecules for the treatment of FTD-GRN. Available: https://www.arkudatx.com/wp-content/uploads/2020/04/EPoster_AATADPD-2020_ArkudaTx.pdf [Accessed 21 Jul 2020].

30 Molgaard S, Demontis D, Nicholson AM, et al. Soluble sortilin is present in excess and positively correlates with progranulin in CSF of aging individuals. Exp Gerontol 2016;84:96-100.

31 Ghidoni R, Flocco R, Paterlini A, et al. Secretory leukocyte protease inhibitor protein regulates the penetrance of frontotemporal lobar degeneration in progranulin mutation carriers. J Alzheimers Dis 2014;38:533-9.

32 van der Ende EL, Meeter LH, Stingl C, et al. Novel CSF biomarkers in genetic frontotemporal dementia identified by proteomics. Ann Clin Trans/ Neurol 2019;6:698-707

33 Barschke $\mathrm{P}$, OeckI P, Steinacker P, et al. Different CSF protein profiles in amyotrophic lateral sclerosis and frontotemporal dementia with C9orf72 hexanucleotide repeat expansion. J Neurol Neurosurg Psychiatry 2020;91:503-11.

34 Bright F, Werry EL, Dobson-Stone C, et al. Neuroinflammation in frontotemporal dementia. Nat Rev Neurol 2019;15:540-55. 
35 Heywood WE, Hallqvist J, Heslegrave AJ, et al. CSF pro-orexin and amyloid- $\beta 38$ expression in Alzheimer's disease and frontotemporal dementia. Neurobiol Aging 2018;72:171-6.

36 Woollacott IOC, Nicholas JM, Heslegrave A, et al. Cerebrospinal fluid soluble TREM2 levels in frontotemporal dementia differ by genetic and pathological subgroup. Alzheimers Res Ther 2018:10:79.

37 Woollacott I, Nicholas J, Heller C, et al. Cerebrospinal fluid YKL-40 and chitotriosidase levels in frontotemporal dementia. Dement Geriatr Cogn Disord 2020:1-21.

38 Abu-Rumeileh S, Steinacker P, Polischi B, et al. CSF biomarkers of neuroinflammation in distinct forms and subtypes of neurodegenerative dementia. Alzheimers Res Ther 2019;12:1-15.

39 Oeckl P, Weydt P, Steinacker $P$, et al. Different neuroinflammatory profile in amyotrophic lateral sclerosis and frontotemporal dementia is linked to the clinical phase. J Neurol Neurosurg Psychiatry 2019;90:4-10.

40 Ishiki A, Kamada M, Kawamura Y, et al. Glial fibrillar acidic protein in the cerebrospinal fluid of Alzheimer's disease, dementia with Lewy bodies, and frontotemporal lobar degeneration. J Neurochem 2016;136:258-61.

41 Heller C, Foiani MS, Moore K, et al. Plasma glial fibrillary acidic protein is raised in progranulin-associated frontotemporal dementia. J Neurol Neurosurg Psychiatry 2020;91:263-70.

42 Galimberti D, Bonsi R, Fenoglio C, et al. Inflammatory molecules in frontotemporal dementia: cerebrospinal fluid signature of progranulin mutation carriers. Brain Behav Immun 2015:49:182-7.

43 Gibbons L, Rollinson S, Thompson JC, et al. Plasma levels of progranulin and interleukin-6 in frontotemporal lobar degeneration. Neurobiol Aging 2015;36:1603. e1-1603.e4.

44 Galimberti D, Schoonenboom N, Scheltens P, et al. Intrathecal chemokine levels in Alzheimer disease and frontotemporal lobar degeneration. Neurology 2006:66:146-7.

45 Hu WT, Chen-Plotkin A, Grossman M, et al. Novel CSF biomarkers for frontotemporal lobar degenerations. Neurology 2010;75:2079-86.

46 Bossù $P$, Salani $F$, Alberici $A$, et al. Loss of function mutations in the progranulin gene are related to pro-inflammatory cytokine dysregulation in frontotemporal lobar degeneration patients. J Neuroinflammation 2011;8:65

47 Galimberti D, Venturelli E, Fenoglio C, et al. Intrathecal levels of IL-6, IL-11 and LIF in Alzheimer's disease and frontotemporal lobar degeneration. J Neurol 2008;255:539-44.

48 Rentzos M, Paraskevas GP, Kapaki E, et al. Interleukin-12 is reduced in cerebrospinal fluid of patients with Alzheimer's disease and frontotemporal dementia. J Neurol Sci 2006;249:110-4.

49 Rentzos M, Zoga M, Paraskevas GP, et al. IL-15 is elevated in cerebrospinal fluid of patients with Alzheimer's disease and frontotemporal dementia. J Geriatr Psychiatry Neurol 2006:19:114-7.

50 Sjögren M, Folkesson S, Blennow K, et al. Increased intrathecal inflammatory activity in frontotemporal dementia: pathophysiological implications. J Neurol Neurosurg Psychiatry 2004;75:1107-11.

51 Starhof C, Winge K, Heegaard NHH, et al. Cerebrospinal fluid pro-inflammatory cytokines differentiate parkinsonian syndromes. J Neuroinflammation 2018;15:305.

52 Miller ZA, Rankin KP, Graff-Radford NR, et al. TDP-43 frontotemporal lobar degeneration and autoimmune disease. J Neurol Neurosurg Psychiatry 2013;84:956-62.

53 Lui $\mathrm{H}$, Zhang J, Makinson SR, et al. Progranulin deficiency promotes circuit-specific synaptic pruning by microglia via complement activation. Cell 2016;165:921-35.

54 Yamada T, Moroo I, Koguchi Y, et al. Increased concentration of C4d complement protein in the cerebrospinal fluids in progressive supranuclear palsy. Acta Neurol Scand 1994;89:42-6.

55 Fraga VG, Magalhães CA, Loures CdeMG, et al. Inflammatory and pro-resolving mediators in frontotemporal dementia and Alzheimer's disease. Neuroscience 2019;421:123-35.

56 Skillbäck T, Delsing L, Synnergren J, et al. CSF/serum albumin ratio in dementias: a cross-sectional study on 1861 patients. Neurobiol Aging 2017;59:1-9.

57 Janelidze S, Hertze J, Nägga K, et al. Increased blood-brain barrier permeability is associated with dementia and diabetes but not amyloid pathology or APOE genotype. Neurobiol Aging 2017;51:104-12.
58 Hansson O, Santillo AF, Meeter LH, et al. CSF placental growth factor - a novel candidate biomarker of frontotemporal dementia. Ann Clin Trans/ Neurol 2019;6:863-72.

59 Morelli C, Tiloca C, Colombrita C, et al. CSF angiogenin levels in amyotrophic lateral Sclerosis-Frontotemporal dementia spectrum. Amyotroph Lateral Scler Frontotemporal Degener 2020;21:63-9.

60 Mahlknecht P, Stemberger S, Sprenger F, et al. An antibody microarray analysis of serum cytokines in neurodegenerative parkinsonian syndromes. Proteome $\mathrm{SC}$ 2012;10:71.

61 Tuna G, Yener GG, Oktay G, et al. Evaluation of matrix metalloproteinase-2 (MMP-2) and -9 (MMP-9) and their tissue inhibitors (TIMP-1 and TIMP-2) in plasma from patients with neurodegenerative dementia. J Alzheimers Dis 2018:66:1265-73.

62 Goetzl EJ, Boxer A, Schwartz JB, et al. Altered lysosomal proteins in neural-derived plasma exosomes in preclinical Alzheimer disease. Neurology 2015;85:40-7.

63 Boman A, Svensson S, Boxer A, et al. Distinct lysosomal network protein profiles in parkinsonian syndrome cerebrospinal fluid. J Parkinsons Dis 2016;6:307-15.

64 Parnetti L, Balducci C, Pierguidi L, et al. Cerebrospinal fluid betaglucocerebrosidase activity is reduced in dementia with Lewy bodies. Neurobiol Dis 2009;34:484-6.

65 Clarke MTM, Brinkmalm A, Foiani MS, et al. CSF synaptic protein concentrations are raised in those with atypical Alzheimer's disease but not frontotemporal dementia. Alzheimer's Res Ther 2019;11.

66 Goetzl EJ, Kapogiannis D, Schwartz JB, et al. Decreased synaptic proteins in neuronal exosomes of frontotemporal dementia and Alzheimer's disease. Faseb J 2016:30:4141-8

67 Oeckl P, Halbgebauer S, Anderl-Straub S, et al. Targeted mass spectrometry suggests beta-synuclein as synaptic blood marker in Alzheimer's disease. J Proteome Res 2020;19:1310-8

68 Sáez-Valero J, Costell M, Sjögren M, et al. Altered levels of cerebrospinal fluid reelin in frontotemporal dementia and Alzheimer's disease. J Neurosci Res 2003;72:132-6

69 van der Ende EL, Xiao M, Xu D, et al. Neuronal pentraxin 2: a synapse-derived CSF biomarker in genetic frontotemporal dementia. J Neurol Neurosurg Psychiatry 2020;91:612-21.

70 Engelborghs S, Vloeberghs E, Le Bastard N, et al. The dopaminergic neurotransmitter system is associated with aggression and agitation in frontotemporal dementia. Neurochem Int 2008;52:1052-60.

71 Janssens J, Vermeiren $\mathrm{Y}$, van Faassen $\mathrm{M}$, et al. Monoaminergic and kynurenergic characterization of frontotemporal dementia and amyotrophic lateral sclerosis in cerebrospinal fluid and serum. Neurochem Res 2020;45:1191-201.

72 Grasso M, Piscopo P, Talarico G, et al. Plasma microRNA profiling distinguishes patients with frontotemporal dementia from healthy subjects. Neurobiol Aging 2019;84:240.e1-240.e12

73 Piscopo P, Grasso M, Puopolo M, et al. Circulating miR-127-3p as a potential biomarker for differential diagnosis in frontotemporal dementia. J Alzheimers Dis 2018:65:455-64.

74 Schneider R, McKeever P, Kim T, et al. Downregulation of exosomal miR-204-5p and miR-632 as a biomarker for FTD: a GENFI study. J Neurol Neurosurg Psychiatry 2018;89:851-8.

75 Murley AG, Jones PS, Coyle Gilchrist I, et al. Metabolomic changes associated with frontotemporal lobar degeneration syndromes. J Neurol 2020;267:2228-38.

76 Santos ALM, Vitório JG, de Paiva MJN, et al. Frontotemporal dementia: plasma metabolomic signature using gas chromatography-mass spectrometry. J Pharm Biomed Anal 2020;189:113424.

77 Mori A, Ishikawa K-I, Saiki S, et al. Plasma metabolite biomarkers for multiple system atrophy and progressive supranuclear palsy. PLoS One 2019;14:e0223113.

78 Kim WS, Jary E, Pickford R, et al. Lipidomics analysis of behavioral variant frontotemporal dementia: a scope for biomarker development. Front Neurol 2018:28.

79 Phan K, He Y, Pickford R, et al. Uncovering pathophysiological changes in frontotemporal dementia using serum lipids. Sci Rep 2020;10:3640.

80 Jääskeläinen 0 , Solje $E$, Hall A, et al. Low serum high-density lipoprotein cholesterol levels associate with the C9orf72 repeat expansion in frontotemporal lobar degeneration patients. J Alzheimers Dis 2019;72:127-37. 